\title{
Ultrasound Imaging Simulation: Application to the Diagnosis of Deep Venous Thromboses of Lower Limbs
}

\author{
D. Henry*, J. Troccaz*, J.L. Bosson+ and O. Pichot+ \\ *TIMC Laboratory - IAB, Faculté de Médecine de Grenoble \\ 38706 La Tronche, France \\ +Service Médecine interne et Angiologie - CHU Grenoble \\ 38043 Grenoble Cedex, France \\ Jocelyne.Troccaz@imag.fr
}

\begin{abstract}
This paper describes an ultrasound imaging simulator dedicated to the training of physicians for the detection of deep venous thromboses of the lower limbs. Currently, a lot of pathologies of soft tissue are readily diagnosed using ultrasound imaging. This is the case of deep venous thromboses of the lower limbs. Because this examination is difficult and operator-dependent, developing a simulator is very useful to give common databases of pathological samples on which physicians can both experiment image acquisition and evaluate their understanding of clinical cases. An ultrasound imaging simulator has been developed. An ultrasound volume is constructed from real images of any typical patient in an off-line pre-processing. Then, simulated images are generated from this volume. The image generation takes into account both the position of the virtual probe and the pressure applied by this probe on the body. Virtual ultrasonic images are generated using a particular interpolation technique and a deformable model of the significant anatomical structures. In most cases, the simulated images are indistinguishable from real ultrasound images.
\end{abstract}

\section{$1 \quad$ Introduction}

Echography has become one of the preferred mean of diagnosis in several pathologies of soft tissues. This comes mainly from its low cost, and non invasiveness. However, compared to conventional radiology, it does not permit later re-reading which raises a major problem for training, evaluation and for differed discussion of cases. Moreover, diagnosis from echographic images may be difficult because images are often of poor quality. Part of the image understanding comes from the dynamic character of the acquisition. This dynamic aspect is important both in terms of probe position and reaction of the anatomical structures. This reaction results from the pressure exerted by the probe over the body. Regarding diagnosis of thromboses, it is generally considered that the number of necessary examinations before having a good technical competence is around 1000. The first 500 examinations made by a student have to be supervised by a senior 
physician. The possibility of virtual echography, providing intensive training of students. Thus, they could train themselves to acquire images and evaluate their understanding of clinical cases. The virtual echography would allow an increase in the extensive training skills including rare cases, independently of the number of patients of a given medical department. Besides, such a virtual echographic system could speed up new treatment evaluation since it would allow to establish evaluation standards [8].

This paper introduces a computer-based simulator designed to train physicians using ultrasound systems in the diagnosis of deep venous thromboses. In the following sections, we present the medical context of the study (section 2) and an overview of different approaches which can be considered in order to generate virtual ultrasonic images (section 3). In section 4, we present our technical approach and describe the image generation (interpolation and deformation methods). Results are compared to real ultrasound images in section 5 . We conclude in section 6 .

\section{Medical protocol}

Our study focuses on the simulation of echographic acquisitions for the diagnosis of deep venous thromboses of the thigh. Ultrasound examination for deep venous thrombosis detection consists in scanning the whole deep venous network perpendicular to vessels (Figure 1). An increasing pressure is applied by the probe on the thigh to observe the vein deformation in the ultrasonic images. From this behaviour, one can decide whether or not a vessel is partly or totally obstructed without necessarily directly seeing a blood clot on a static image.
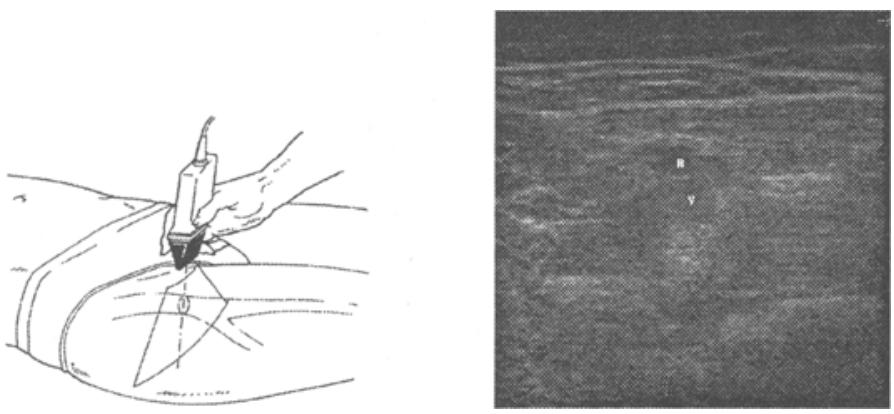

Fig. 1. Examination position (left) and typical ultrasonic image (right)

Deep venous thromboses of lower limbs can be detected by analyzing the vein collapsing when applying increasing pressure on the thigh with the echographic probe. Figure 2 sketches out different echographic images that would be obtained by applying increasing pressure in a non-pathological case. The typical image sequence observed along the arterio-venous axis during the examination is the 


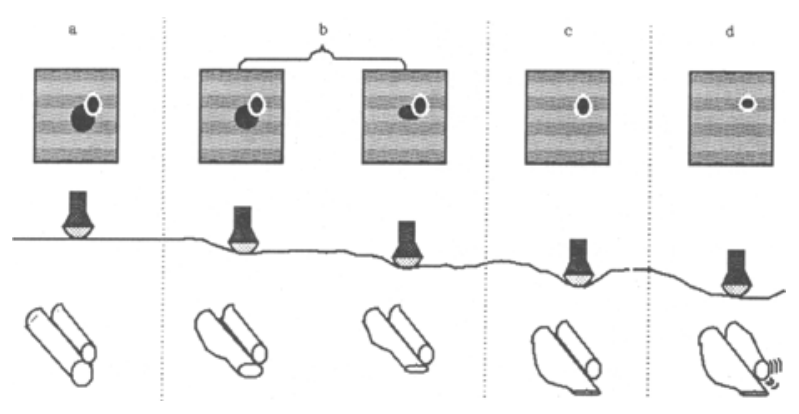

Fig. 2. Echographic aspect of a non pathological vein

following. Vein in relaxation during the initial situation where there is no exerted pressure (cf. fig. 2.a). Figure 1 shows a typical image echographic image of the thigh in state a. Progressive flattening of the vein as the pressure increases (cf. fig. 2.b). Complete venous collapsus when probe pressure is bigger than veinal blood pressure. The vein disappears from the image (cf. fig. 2.c). Arterial hyperpulsatility occurs if probe pressure goes over arterial pressure (cf. fig. 2.d).

Vein incompressibility, detected from the image deformation, is the pathological sign of a thrombosis. In a pathological case, the image would remain in the state described in figure 2.b or somewhere between the states b and c. When arterial hyperpulsatility is reached (state d), the vein may collapse even if obstructed. This state should be avoided as much as possible to avoid both wrong diagnosis and the effect of potential motion of a blood clot in the venous tree.

\section{Existing approaches for the simulation of ultrasound imaging}

The simulation process can be split into a visual and a gestural part. Existing simulators focus on the visual part of this process. It concerns the real-time generation of realistic images to be displayed on a screen from the position of the probe relatively to the anatomical structures. Two approaches can be considered to generate such images.

Ultrasound imaging is based on ultrasonic wave propagation inside matter. The generative approach is a physical approach consisting in modelling this propagation. Therefore, in order to generate images, we need an accurate model of the probe, a description of organs to be observed and a model of the interaction between the ultrasonic wave and the tissues. This is a very complex problem which has been tackled in several ways depending on the objectives of the simulation (cf. [7], [2], [5], [11]). Generative approaches have the avantage of being applicable to any type of organs provided that a model of them is available. However, the phenomena which have to be modelled are extremely complex and time-consuming. In our context where real-time simulation is needed, a large 
computational time is totally prohibitive. The compromise which has been proposed by $\mathrm{E}$. Varlet reduces the computing time but does not allow yet to generate textured images.

The second approach is based on the acquisition of a more or less dense volume of ultrasonic data from which new images can be computed (see [1], [3]). The 3D volume of data is acquired in an off-line preprocessing and images are generated during simulation, by computing a slice in the volume. Such images can be generated very rapidly. As they come from real ultrasound images, there is no realism problem.

As stated previously, ultrasonic imaging is a dynamic examination. Images does not only depend on the probe position and orientation but also on the pressure exerted onto the body. The simulation has to take into account deformations induced by this pressure. Very few attention has been paid to this problem in the existing simulators. UltraSim [1] includes elastic registration tools to build a coherent volume from data acquired under different probe positions therefore involving different local deformations. Nevertheless, during simulation no deformation is applied onto the images when the computed pressure of the virtual probe varies. Varlet [11] proposes a local non-linear model of deformation of the computed image allowing to deal with the constraints applied by the tip of the echo-endoscope to the local tissues.

\section{Our approach}

We have chosen an approach based on the computation of virtual images from a 3D ultrasound data volume. This approach seems to be well-suited to our requirements: realism and real-time. Also for real-time reasons, a deformation model operating on 2D images without pressure consideration has been selected. The system works has follows. Let consider a pathological case of interest. A volume of 3D ultrasonic data is acquired with no significant pressure for database construction. The ultrasound probe is instrumented with infrared diodes which position is tracked using an optical localizer. Using this system, the position and orientation of the echographic plane can be recorded simultaneously to the image. No specific constraints on the position and orientation of the images apply during data acquisition. Regions with thromboses are localized using the protocol described previously and the corresponding images with pressure are also recorded with their position. These sets of images are segmented to compute 3D surfaces of the relevant anatomical structures (cf. [4]) with and without pressure. During simulation, after initialization, the position of the virtual probe is tracked by a localizing device. From the detected position of the virtual echographic plane, a first image is computed using an interpolation method with no consideration of pressure. This image is deformed in a second step to take pressure into account. This second image is displayed to the user. 


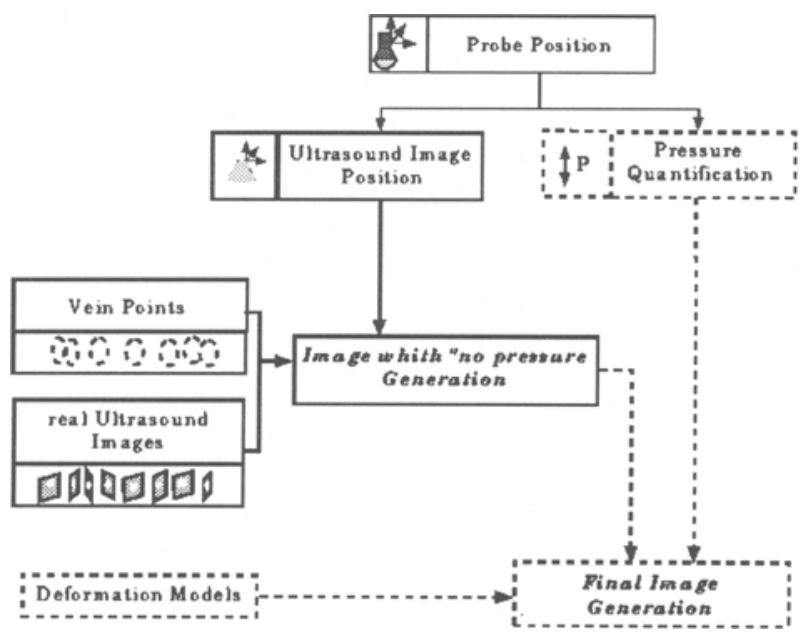

Fig. 3. Image generation

\subsection{Interpolation}

As already mentioned, firstly, an ultrasound image with no pressure consideration has to be generated. This requires the implementation of an interpolation method which is able to reconstruct an image in a given position from a $3 \mathrm{D}$ ultrasound data set. Images from this set have any orientations. Therefore, it is difficult to find an interpolation direction. Due to the image poor quality, it is also very difficult to use interpolation methods such as the shape-based interpolation [9]. Discontinuities due to speckle and to our volume low-density do not allow to use classical interpolation methods for instance using a spline function of the grey level over the volume of data. In order to deal with these problems we have chosen to develop a new interpolation method; we have tested different methods which common point is to focus on the vein principal direction. Because deep venous thrombosis examination is mainly based on vein image observation, its looks reasonable to take the vein as our interpolation direction.

The developed method has been inspired by J. Lengyel's work on 3D reconstruction of intravascular ultrasound slices [6]. Let consider the spline curve $C$ interpolating the centers of the vein in the acquired images. The interpolation method consists in firstly transforming the initial reference system related to the vein (represented by the $C$ curve) into an orthogonal reference system where the vein is represented as a line $D$ (cf. figure 4). In this new space, images can be generated using a linear interpolation method on pixel grey levels: each pixel of the transformed images is linked to its two closest neighbours along $D$ in a suitable database. For each pixel of the synthesized image, the grey intensity is obtained very rapidly by linear interpolation of the closest pixels found in the database. 


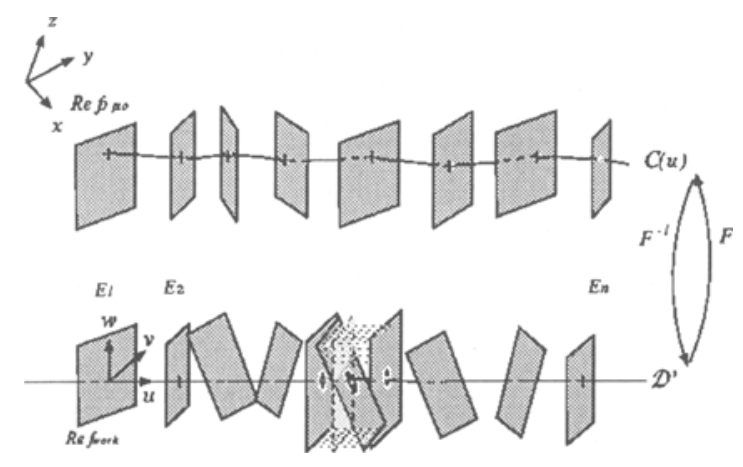

Fig. 4. From a curval reference system to an orthogonal reference system

\subsection{Deformation}

To represent the deformation due to the probe pressure, we need to find a model which input is this $2 \mathrm{D}$ image with no pressure consideration and which output is another $2 \mathrm{D}$ image taking into account tissue deformation due to the probe pressure. The main difficulty of this modelling lies in the fact that each structure has its own way to react under pressure. Arteries have almost no deformation. Non pathological veins flatten. Superficial soft tissues have a linear deformation. Deep tissues have almost no deformations. Due to these differences we can not apply the same deformation to the whole image. We have tested a first method which involved different functions of deformations depending on the position of the pixel into these four types of structures. The major problem we had to face lied in the discontinuities obtained in the synthetic image at the boundaries of the structures. Therefore, we propose a more general deformation model based on the elastic registration of images acquired at different levels of pressure. The idea is to deform the $2 \mathrm{D}$ image using an elastic transformation computed from relevant anatomical contours. The process is the following (cf. figure 5) :

- Selection of the sets of points to be matched : in our case, the vein, the artery and the skin surface. This provides two sets of $2 \mathrm{D}$ points. They are computed from two pre-processed 3D models of the structures of interest (with and without pressure) (cf. [4]).

- Computation of the non-rigid transformation between these sets of points : we propose to represent this transformation using octree-splines (here a quadtree-spline) as described in [10].

- Image generation : A regular grid is associated to the "pressure image" that has to be computed. It is transformed by the elastic transform found in the previous stage into another irregular grid super-imposed to the "non-pressure image" computed using the interpolation methods described previously. Each pixel from the regular grid may be mapped into a pixel or a neighbourhood 
of pixels of the irregular grid. Its grey intensity is deduced from them as the mean value.

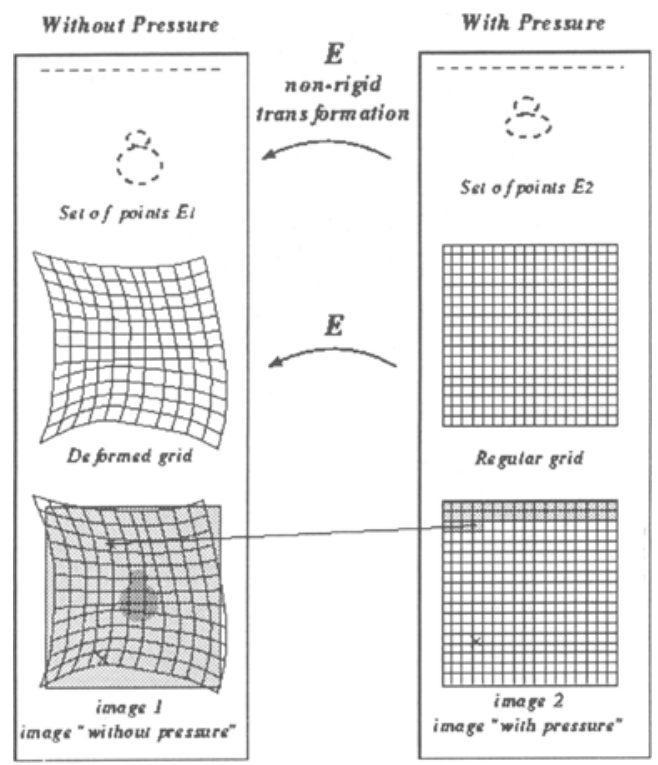

Fig. 5. Image generation including deformations induced by pressure exerted by the probe over the body

\section{Results}

Interpolation and deformation methods have been implemented and tested in real conditions. The different interpolation methods have been tested using both synthetic and real 3D ultrasound data sets. Given those results, we have chosen the presented interpolation method. To compare real images to virtual ones, we have decided to test our methods at a real acquisition position. The real ultrasound image is removed from the $3 \mathrm{D}$ ultrasound data set. Then for this position, an ultrasound image is generated using our interpolation method. The result can be seen on the figure 6 . Another image has been generated at an arbitrary position in the ultrasound volume as illustrated on figure 7).

To test the selected deformation method, we have generated an ultrasound image corresponding to a really acquired image. The pressure which has been selected corresponds to a medium pressure for which the vein is still visible in the image. Results can be seen Figure 8 . 

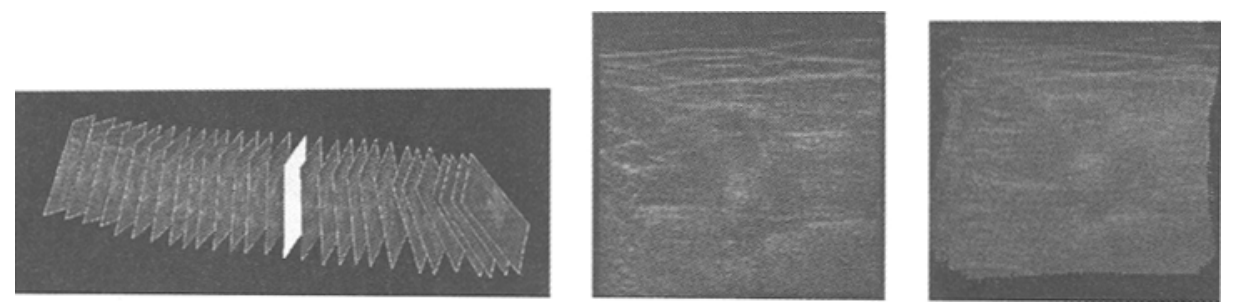

Fig. 6. (left) Ultrasound images from a thigh ultrasound examination (the image to be generated is represented in white) - (middle) Real image - (right) Interpolated image
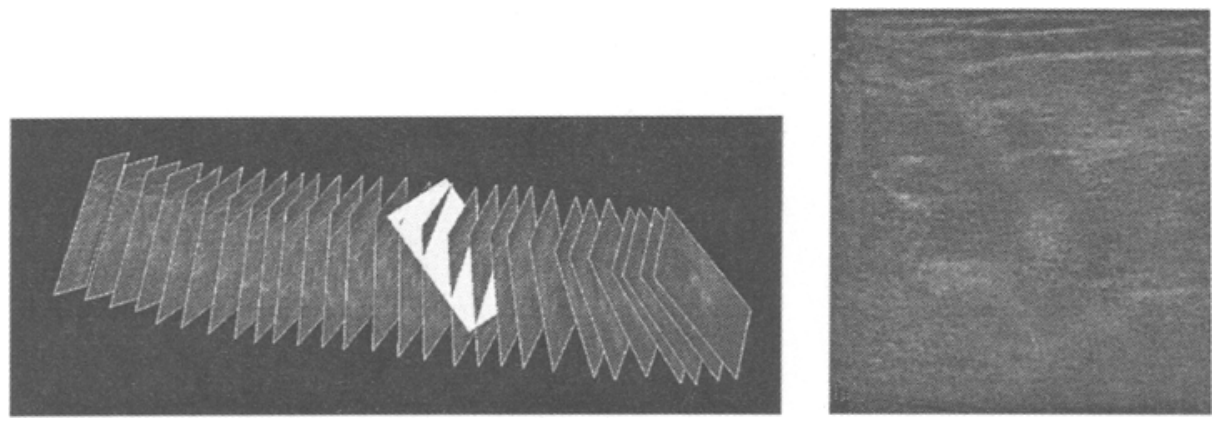

Fig. 7. (left) Ultrasound images from a thigh ultrasound examination. The image to be generated is represented in white. (Right) Virtual image generated by our interpolation method

Concerning realism, virtual images are similar to real ones. Concerning realtime constraint, images ( $512 \times 512$ pixels) have been computed on a DEC Alpha workstation with a $500 \mathrm{Mhz}$ processor. Generating an image with the interpolation method takes about $0.3 \mathrm{~s}$ and deforming it takes less than $0.1 \mathrm{~s}$, if the non-rigid registration is computed in an off-line process.

\section{Conclusion}

This paper has presented different tools necessary to implement a simulator of ultrasound imaging based on a simple and effective concept where a data volume is constructed from real ultrasound images in an off-line process. Then, during the simulation stage, arbitrary slices of the volume are interpolated and postprocessed to simulate ultrasound images rendering deformation phenomena. Interpolated images have been computed and compared to actual images and the results are very promising. Nevertheless, further steps will be necessary to address other pathologies elsewhere in the body and to be able to render force and tactile feeling to the operator. 

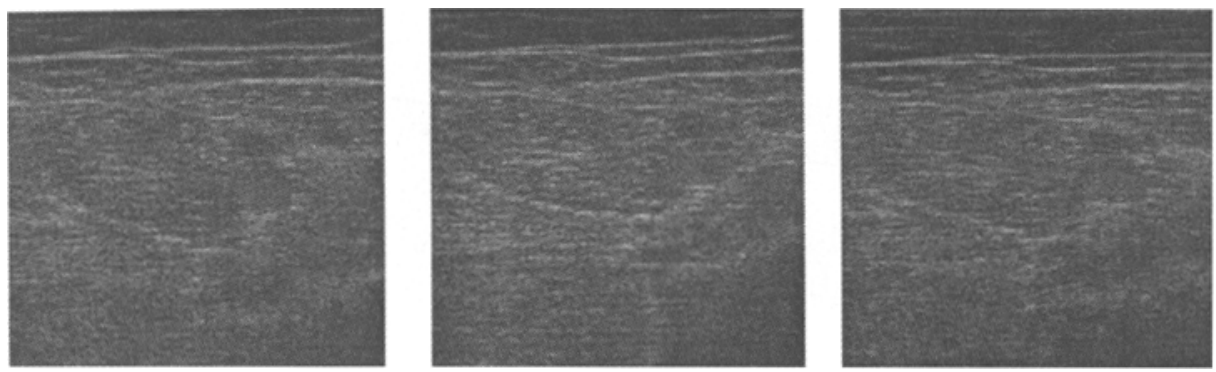

Fig. 8. Generating images with pressure : (left) real image without pressure - (middle) real image with pressure - (right) virtual image synthesized using the deformation model

\section{References}

1. D. AIger AND D. Cohen, Real-time ultrasound imaging simulation, RTI, (1997). trouver sur le web http://www.math.tau.ac.il/ daniel/Default.html.

2. J. BAMBER AND R. DiCKINSON, Ultrasonic B-scanning : a computer simulation, Physics in Medicine and Biology, 25 (1986), pp. 41-60.

3. T. BERLAGE, Augmented reality for diagnosis based on ultrasound images, in CVRMed-MRCAS'97, Springer, ed., 1997, pp. 253-262.

4. G. Champleboux AND D. Henry, Parametric two-dimensional B-Spline representation of vein and artery from 2.5D echography used to aid virtual echography., in CVRMed-MRCAS'97, R. M. J. Troccaz, E.Grimson, ed., LNCS 1205, Springer, 1997, pp. 263-272.

5. J. JENSEN, Field : A program for simulating ultrasound systems, in 10th northbaltic conference on biomedical imaging, 1996, pp. 351-353. trouver sur le web.

6. J. Lengyel, D. Greenberg, A. Yeug, and E. Alderman, Three-dimensional reconstruction and volume rendering of intravascular ultrasound slices imaged on a curved arterial path, in Computer vision, Virtual Reality, and Robotics in Medicine, CVRMed, 1995, pp. $399-405$.

7. J. MEUNIER, Analyse des textures d'échocardiographie bidimensionnelles du myocarde, $\mathrm{PhD}$ thesis, Université de Montreal, avril 1989. in french.

8. O. PICHOT AND AL., An echographical score for the quantification of deep vein thrombosis. Interest in the follow-up examination., Advances in vascular pathology, 3 (1990), p. 471.

9. S. RAYA AND J. UDUPA, Shape based interpolation of Multidimensional objects, IEEE transactions on medical imaging, 9 (1990), pp. 32-42.

10. R. SzelisKI AND S. LAVALleE, Matching 3-D anatomical surfaces with non-rigid deformations using octree-splines, Int. J. of Computer Vision (IJCV), (18) (1996), pp. 171-186.

11. E. VARLET, Etude d'un simulateur pédagogique d'écho-endoscopie digestive, modélisation et réalisation, $\mathrm{PhD}$ thesis, Université Sciences et Techniques de Lille, octobre 1997. 